\title{
Long-term continuous Trastuzumab use for HER2-positive advanced breast cancer
}

\author{
Haider Y. Shukur
}

Najaf Cancer Clinic (NCC), Departments of Oncology, Faculty of Medicine,, Ibn Hayyan Medical University, Najaf, Iraq.

Corresponding Author: Haider Y. Shukur (E-mail: shukurhaider@yahoo.com)

(Submitted: 12 April 2020 - Revised version received: 28 May 2020 - Accepted: 11 June 2020 - Published online: 30 October 2020)

\begin{abstract}
Objectives: Trastuzumab is the standard of care for locally advanced/metastatic her2-positive breast cancer. However, most of these patients will progress within 12 months of trastuzumab therapy. In contrast, there is a paucity of data available on the long-term treatment of patients with Trastuzumab. Our study was conducted to report efficacy and safety data for patients with locally recurrent/metastatic her2-positive breast cancer who received long-term therapy with Trastuzumab ( $\geq 5$ years).

Methods: This study was a prospective single-arm study of continuous Trastuzumab in patients who were histologically her2-positive and radiologically confirmed inoperable locally recurrent or metastatic breast cancer after complete 1 year of Trastuzumab plus chemotherapy (in hormone negative/hormone resistance) treatment then continuous Trastuzumab alone, or with hormone therapy (in sensitive hormone positive) without progression [complete or partial response or stable disease]. A total of 50 inoperable local recurrent and metastatic breast cancer patients were treated with continuous intravenous Trastuzumab therapy administered according to the standard Trastuzumab every 3-weeks ( $8 \mathrm{mg} / \mathrm{kg}$ loading dose followed by 3-weekly $6 \mathrm{mg} / \mathrm{kg}$ maintenance doses starting 3 weeks after the loading dose) schedule, from January 2014 to January 2019 at the Najaf Cancer Clinic.

Results: All 50 patients were evaluated with CR occur only in 20\% (10/50) with an OAR of 50\% (25/50). The cardiac status of these patients remained stable over time for the majority of patients with no marked changes in left ventricular ejection fraction\%. No treatment-related death was observed. The median OS and median PFS is 61 months and 20 months, respectively.

Conclusion: In her2-positive recurrent and metastatic breast cancer patients, who initially respond to palliative treatment with trastuzumab, continuous trastuzumab can achieve a long-term tumor remission of several years and had significantly improved survival with tolerated and acceptable adverse events.
\end{abstract}

Keyword: Trastuzumab, Breast neoplasms, HER2-positive, Iraq

\section{Introduction}

Cancer breast is the utmost frequently identified cancer worldwide and is the principal cause of cancer-linked death in women.

The American Cancer Society estimates that 268,600 Americans will be detected with invasive breast cancer and 41,760 will die in $2019 .{ }^{2}$

About $15 \%-25 \%$ of breast cancers show her- 2 amplification or overexpression (human epidermal growth factor receptor-2). ${ }^{3}$

Every year, at least 276,000 women worldwide receive a recent diagnosis of breast cancer positive for her-2 (1.38 million $\times 20 \%$ ), a number that is estimated to increase to 540,000 by $2030(2.7$ million $\times 20 \%)$. ${ }^{4}$

Although most cancer breast patients can be cured of their disease, up to $20 \%$ will develop metastatic breast cancer (MBC) and it has consistently been considered an incurable disease. ${ }^{5}$

Her2-positive patients with breast cancer previously associated with an unfavorable prognosis, since it is a more aggressive disease and significantly reduces disease-free survival in localized diseases and reduces overall survival in metastatic diseases compared to their counterparts without overexpression. ${ }^{6}$

Conversely, the outcome of patients with her2-positive breast cancer has significantly improved through the use of therapeutic agents targeting her2, such as trastuzumab (herceptin), pertuzumab, ado-trastuzumab emtansine (T-DM1), and lapatinib altered disease outcomes in this subgroup of cancer. ${ }^{7}$

An in-depth analysis of the Epidemiological Strategy and Medical Economics (ESME) database showed a median overall survival of 51.1 months in 2012, compared to 38.7 months in $2008 .^{8}$
The use of trastuzumab has meaningfully altered the ordinary history of her2-positive $\mathrm{MBC}$, translating it from a historically aggressive disease subtype to a subtype with better prognosis and prolonged survival. ${ }^{9}$

Trastuzumab (Herceptin); F. Hoffmann-La Roche Ltd, Basel, Switzerland), a humanized monoclonal antibody against her2, revolutionized the treatment of her2-positive breast cancer that was prime approved in 1998 in the America and 2000 in Europe, for the treatment of MBC with her2-positive. Since then, trastuzumab has been documented in more than 125 nations around the world, leading to a new standard of care for her2-positive disease. ${ }^{10}$

Adjuvant treatment with trastuzumab can reduce the relative risk of recurrence by $46 \%-52 \%$ at 2-3.5 years, and trastuzumab + pertuzumab with combinations of chemotherapy have been displayed to have impressive overall survival in metastatic disease. ${ }^{11}$

Although a large percentage of positive her2 MBC patients treated with trastuzumab recurrence after 12-24 months, few patients have prolonged remission. ${ }^{12}$

Treatment guidelines for metastatic her2-positive patients suggest that her 2 target agents must be involved in therapeutic regimens of multiple therapy lines, as this has been associated with better patient outcomes. ${ }^{13}$

Trastuzumab treatment has been connected with longterm survival, particularly in patients who have remained progression-free for $>2$ years of trastuzumab treatment. ${ }^{14}$

Most studies support maintenance treatment with trastuzumab until progression of the disease or at the request of the 
patient, and this remains the standard after response to firstline chemotherapy and an anti-her 2 agent that in some cases may last many years. ${ }^{15,16}$

The ideal trastuzumab duration for patients who obtain a complete response (CR) is still under debate. Cardiac toxicity is the most significant limiting factor for the use of trastuzumab, although cardiac events are rare and mostly asymptomatic in trastuzumab studies beyond progression. ${ }^{17}$

Although there is a lack of evidence regarding treatment discontinuation in response cases, some small clinical case series characterized patients with her2-positive breast cancer who attained CRs with chemotherapy with trastuzumab-containing regimens, and the patients persisted disease-free without her2 therapy for years. ${ }^{18}$

\section{Patients and Methods}

\section{Patient Eligibility}

This was a prospective study involving patients with her2-positive histology and radiologically confirmed as locally inoperable recurrent or MBC after accomplished 1 year of treatment with trastuzumab plus chemotherapy (hormone negative/hormonal resistance) and then continued trastuzumab, or with hormone therapy (in sensitive hormone positive) without progression [complete or partial response (PR) or stable disease] to continuous trastuzumab received from January 2014 to January 2019 at the Najaf Cancer Clinic (NCC) Affiliated Clinic, Jaber Ibn Hayyan Medical University was eligible for inclusion. In all cases, locally inoperable recurrent or metastatic multidisciplinary breast cancer team was diagnosed according to the definitions of the National Comprehensive Cancer Network .her2-positive status was defined in the primary tumor or in metastatic biopsies as immunohistochemistry (IHC) 3+ staining or 2+ IHC and positive in situ hybridization (SISH) or fluorescence in situ hybridization (FISH, HER2 / CEP17 ratio> 2.2) according to ASCO/CAP guidelines. ${ }^{19}$

\section{Inclusion Criteria}

1) WHO-performance state (WHO-PS) of 0-2.

2) Age from 20 to 75 years.

3) Patients with signs of chronic cardiac failure might be included after an investigator risk-benefit assessment.

4) Patient with solitary kidney was included.

5) Written informed consent has been requested.

\section{Exclusion Criteria}

1) Over 75 years old.

2) Pregnant or lactating women, female of childbearing time unless they use effective contraceptive measures.

3) Severe dyspnea requiring supplemental oxygen therapy and uncontrolled severe systemic illness.

4) Patients with a simultaneous diagnosis of another none breast cancer.

5) Those who have not received first-line trastuzumab.

6) $\mathrm{WHO}-\mathrm{PS} \geq 3$.

7) Abnormal biochemistry (i.e., bilirubin 1.3-ULN, alkaline phosphatase 5-ULN, AST/ALT 5-ULN).

8) Inadequate bone marrow.

\section{Pre-Treatment Evaluation Included}

All patients were assessed at baseline and then every four doses of trastuzumab (every 3 months) using chest X-ray and high-resolution chest abdomen and pelvic computed tomography (HRCT) scan, abdominal and pelvic ultrasound. Serum cancer antigen (CA15-3) \& carcinoembryonic antigen (CEA) levels were analyzed for pretreatment as a basis and at interval when evaluating tumor response every 3 months.

Physical examination and laboratory tests $(\mathrm{CBC} \&$ biochemistry) were performed each time before treatment. The tumor response was classified into CR, PR, stable disease (SD) and progressive disease (PD) according to the criteria to estimate the response in solid tumors (version 1.1) mentioned by Eisenhauer et al.12

Adverse events were classified according to the common terminological criteria of the NCI for serious adverse events (version 4.0). Overall survival (OS) was distinct as the time concerning to the start of trastuzumab maintenance \& death date. Progression-free survival (PFS) was recognized as the time between the start of trastuzumab maintenance and the first progression and/or death. Patients censored are those without an event on the last follow-up date (February 1, 2020). For patients with continuous trastuzumab, information was collected at the time of hospitalization.

Patients withdrawn from the study in case of intolerable toxic effects or evidence of disease progression, or at their request, move to another line or best supportive care accordingly. For those patients with SD or PR, the duration of trastuzumab continuous until study end.

\section{Treatment Schedule}

Patients formerly treated with trastuzumab therapy sustained to receive intravenous trastuzumab therapy ordered according to the trastuzumab standard program every 21 days $(8 \mathrm{mg} / \mathrm{kg}$ loading dose then continuous by every 21 days $6 \mathrm{mg} / \mathrm{kg}$ maintenance doses). Trastuzumab therapy was continued until progression of the disease, intolerable toxicity, patient choice, patient death, or study end. Administration of trastuzumab may be delayed in the case of cardiac toxicity by following an algorithm constructed on evaluation of the left ventricular ejection fraction (LVEF), but no dose adjustments have been allowed. If delayed, trastuzumab is restarted according to the reload guidelines. For patients whose dose was delayed by $\leq 14$ days, a maintenance dose was ordered as soon as possible and consequent maintenance doses were sustained according to primary program. For those whose dose was postponed $>14$ days, a loading dose of $8 \mathrm{mg} / \mathrm{kg}$ was administered, and then maintenance doses were reinitialized and administered continuous by every 21 days. The CBC \& other necessary blood tests were performed regularly every 3 weeks before each dose.

\section{Treatment Evaluations}

During treatment, assessment, including toxicity assessment and response:

Treatment evaluations for disease progression were performed as clinically indicated and as previously described \& the date of disease progression was recorded.

Toxicity Assessments: Cardiac status was assessed by echocardiogram every 4 doses (every 3 months) and as clinically directed. Heart failure (New York Heart Association [NYHA] class II - IV) was reported as a serious adverse event (SAE) and classified according to the criteria of NCI-CTCAE version 3 or NCI-CTC version 2 and the NYHA classification. SAEs have been reported in accordance with the International Council 
for Harmonization (ICH) Guidelines for the Management of Clinical Safety Data, Definitions and Standards for Expedited Reporting. Hematology and biochemistry laboratory evaluations were performed according to standard medical practice.

The main objective of the present study was to evaluate the overall survival (OS) of patients with locally non-operable recurrent and MBCs her2-positive who had a long-term response with continuous trastuzumab. Secondary goals were progression-free survival and safety.

\section{Statistical Analysis}

The analyzing of the statistics by IBM SPSS Statistics V22.0. Numerical information were expressed as median and range as appropriate. Qualitative facts had been expressed as a percentage. The survival curves have been estimated the usage of the Kaplan-Meier technique.

\section{Results}

Between January 2014 and January 2019, 50 patients were enrolled in our clinic (NCC) and received trastuzumab treatment with a median follow-up of 60 months (range 4-73 months). The basic patient characteristics listed in Table 1 . The age group was between 20 and 75 years old, with an average age of 52 years, while 7 (14\%) were 65 years \& older. Forty-four (88\%) of our patients were women, while only six (12\%) were men. Fourteen (29.7\%) of our patients were still pre-menopaused, while $33(70.3 \%)$ were post-menopausal. Overall, the majority of $45(90 \%)$ of our patients had a good WHO-PS of 0-1 at the start of trastuzumab therapy, while $5(10 \%)$ patients had a WHO-PS of 2 . One-fifth (20\%) of our patients were underweight with a BMI (body mass index $\leq 18$, while more than half $(56 \%)$ of our patients had a normal BMI and the remaining $12(24 \%)$ had overweight with BMI $>25$. Most of the patients (86\%) had ductal histology and 4 (8\%) of the lobular type while remaining 3 patients $(6 \%)$ of other histology. The tumors were generally aggressive, with $30(60 \%)$ patients with grade III tumors and 37 (74\%) with high-level Ki-67\% staining (>30\%). Thirty $(60 \%)$ of our patients were positive hormones, while 20 (40\%) were double negative hormones. At the time of disease recurrence or onset of metastatic disease, the minority of $8(16 \%)$ of our patients suffered from inoperable recurrent locoregional disease, while the majority of $42(84 \%)$ were metastasis. Mostly bone metastases found in 17 (34\%), liver $13(26 \%)$, and lung $9(18 \%)$. Brain metastases were present in only three patients (6\%). Almost one-fifth of patient $(20 \%)$ had relevant comorbidities, which were mainly hypertension (16\% of all patients) or cardiac arrhythmia ( $4 \%$ of all patients). Detailed patient characteristics are listed in Table 1.

Baseline tumor marker results including CA15-3, CEA are also listed in Table 2 . Forty-one patients $(82 \%)$ were found with elevated CA15-3 levels before start trastuzumab, but only nine patients (18\%) had normal CA15-3. On the other hand, only 11 patients (22\%) found with normal baseline CEA, but the remaining 39 patients (78\%) had elevated CEA. The detailed patient characteristics are listed in Table 2.

\section{Treatment Delivery}

In the majority of $30(60 \%)$ patients, trastuzumab was started with continuation of treatment in combination with endocrine therapy, and for the remaining $40 \%$ of patients, trastuzumab

\section{Table 1. Patient clinicopathological characteristics.}

Mre
Post

Pre
Post

ECOG-PS-No.(\%)

0

1

2$$
\text { BM }
$$

\section{$\leq 18$}

$18-25$

$>25$

Histological type

IDC

ILC

Other

Grading

G

G2

$\mathrm{G} 3$

\section{Ki-67 Status-No. (\%)}

Low $(5 \%-15 \%)$

Intermediate (16\%-30\%)

High (>30\%) 37 (74)

Hormone Status- No.(\%)

$\mathrm{ER}+/ \mathrm{PR}+$ (double positive) $20(40)$

ER+/PR- $\quad 7(14)$

ER-/PR+ $3(6)$

ER-/PR-(double negative) $20(40)$

HER2 Status- No.(\%)

$\mathrm{HC}+3$

$9(18)$

Primary tumour size (T) -No.(\%)

$\begin{array}{ll}\text { T0-1 } & 8(16) \\ \text { T2 } & 12(24) \\ \text { T3 } & 13(26) \\ \text { T4 } & 17(34) \\ \text { Primary nodal status (N) -No. (\%) } & \\ \text { N0 } & \\ \text { N1 } & 10(20) \\ \text { N2 } & 7(14) \\ \text { N3 } & 15(30) \\ & 18(36) \\ \text { Disease status before initiation of trastuzumab } & \\ \text { treatment -No. (\%) } & \\ \text { In operable Local Recurrence } & 8(16) \\ \text { 1. Chest wall } & 3(6) \\ \text { 2. Breast } & 1(2) \\ \text { 3. Axillary LN } & 1(2) \\ \text { 4. Supraclavicular LN } & 3(6) \\ \text { Distant Metastasis } & 42(84) \\ \text { 1. Bone } & 17(34) \\ \text { 2. Liver } & 13(26) \\ \text { 3. Lung } & 9(18) \\ \text { 4. Brain } & 3(6) \\ \text { Relevant comorbidities } & 10(20 \%) \\ \text { Hypertension } & 8(16 \%) \\ \text { Cardiac arrhythmia } & 2(4 \%)\end{array}$




\begin{tabular}{|c|c|c|c|c|}
\hline Tumor marker & $0-35 \mathrm{U} / \mathrm{mL}$ & $36-500 \mathrm{U} / \mathrm{mL}$ & $501-1000 \mathrm{U} / \mathrm{mL}$ & More than $1000 \mathrm{U} / \mathrm{mL}$ \\
\hline CA15-3[Pts. No.- (\%)] & $7(14 \%)$ & $24(48 \%)$ & $11(22 \%)$ & $8(16 \%)$ \\
\hline \multirow[t]{2}{*}{ CEA [Pts. No.- (\%)] } & $0-3 \mathrm{ng} / \mathrm{mL}$ & $4-100 \mathrm{ng} / \mathrm{mL}$ & $101-500 \mathrm{ng} / \mathrm{mL}$ & More than $500 \mathrm{ng} / \mathrm{mL}$ \\
\hline & $6(12 \%)$ & 23 (46 \%) & 16 (32 \%) & 5 (10 \%) \\
\hline
\end{tabular}

was continued alone. In the case of endocrine therapy in combination with trastuzumab, the majority of patients $23(46 \%)$ received an aromatase inhibitor and 7 (14\%) received tamoxifin with and without Zoladex.

The median duration of trastuzumab treatment in patients with breast cancer was 3.1 years with an interval from 3 months to 6.0 years. The number of trastuzumab cycles ranged from 4 to 97 cycles with a median of 50 cycles. Postponement of treatment was present in 17 (34\%) patients. The duration of the treatment delay varied from 3 to 10 days with a median of 5 days. Only five (10\%) patients on delayed treatment require hospitalization and the duration varies from 2 to 7 days with a median of 4 days.

At the end of this study, all patients included in this analysis have withdrawn from study treatment. Reasons for withdrawal included the occurrence of serious adverse events (n $=5 ; 10 \%)$; disease progression $(\mathrm{n}=13 ; 26 \%)$; and treatment refused $(\mathrm{n}=2 ; 4 \%)$. Other reasons for discontinuation were reported for 28 patients (56\%), study closure, and only 2 (4\%) patients agreement to discontinue treatment until further disease progression. (Table 3 ).

\begin{tabular}{lc}
\hline Table 3. Reasons for study withdrawal. \\
\hline $\begin{array}{lc}\text { Reason for study withdrawal, } \\
\mathbf{n}(\%)\end{array}$ & $\begin{array}{c}\text { Trastuzumab continuous treatment } \\
\mathbf{N}=\mathbf{5 0}\end{array}$ \\
\hline Serious adverse events & $5(10 \%)$ \\
Disease Progression & $13(26 \%)$ \\
Refusal of treatment & $2(4 \%)$ \\
$\begin{array}{l}\text { Treatment discontinued until } \\
\text { further disease progression }\end{array}$ & $2(4 \%)$ \\
Study closure & $28(56 \%)$ \\
\hline
\end{tabular}

\begin{tabular}{|c|c|}
\hline Variable & Patients No. $=\mathbf{5 0}$ \\
\hline $\begin{array}{l}\text { Median duration of trastuzumab } \\
\text { treatment (years)-range }\end{array}$ & 3.1 years $(0.25-6.0)$ \\
\hline $\begin{array}{l}\text { Median No. of trastuzumab cycles } \\
\text { (range) }\end{array}$ & $50(4-97)$ \\
\hline $\begin{array}{l}\text { Median duration of treatment delay in } \\
\text { days (range) }\end{array}$ & $5(3-10)$ \\
\hline Patient with treatment delay no. (\%) & $6(12 \%)$ \\
\hline $\begin{array}{l}\text { Patient with delayed treatment and } \\
\text { need hospitalization no. (\%) }\end{array}$ & $5 / 17(30 \%)$ \\
\hline $\begin{array}{l}\text { Median duration of hospitalization in } \\
\text { days (range) }\end{array}$ & $4(2-7)$ \\
\hline
\end{tabular}

\section{Safety}

LVEF\% measurements were available for all patients (100\%) included in these analyzes. The cardiac status of these patients remained stable over time for 33 (66\%) patients without marked changes in LVEF\% (range 50-71\%). Twelve (24\%) patients showed a reduction in LVEF\% to less than $50 \%$ (range $47-50 \%)$, but the remaining five (10\%) showed a marked decrease in $\mathrm{LVEF} \%<45 \%$ and developed cardiac toxicity that was not corrected with treatment support, and/or delayed in trastuzumab, therefore trastuzumab cancelled permanently.

Nine $(18 \%)$ of the 50 patients reported skeletal adverse events, but none of them were related to trastuzumab treatment rather than to hormone therapy and menopause. The reported skeletal side-effects were pain, septic arthritis, fibula fracture, rib fracture, and femur fracture, all of which occurred in one patient $(2 \%)$ each. There were no patients who died during study treatment.

\section{Efficacy}

A clinical response to trastuzumab treatment was evaluated every 3 months and was observed in the majority (74\%) of patients with $10(20 \%)$ with complete remission, 15 (30\%) partial remission, and 12 (24\%) stable disease as the greatest response.

A remission (complete or partial) was documented after an average period of 6 months from the continuous start of treatment. Unfortunately, until the end of the study, disease progression occurred in 13 (26\%) patients during continuous trastuzumab treatment with an estimated mean duration of treatment 3.1 years (95\%CI: $0.25-6.0$ years). PFS was estimated to be 20 months (95\% CI: 17.7-22.2 months) and OS 61 months (95\% CI: 51.4-70.5 months). It has been estimated that $82 \%$ of patients remained in remission for 24 months, $50 \%$ for 48 months, and 20\% for up to 70 months. (Table 5, Figs 1 and 2).

Table 5. Objective response rate and survival outcome, №. $=\mathbf{5 0}$.

\begin{tabular}{ll}
\hline Response & No. of patients (\%) \\
\hline Complete remission(CR) & $10(20 \%)$ \\
Partial Response (PR) & $15(30 \%)$ \\
Stable Disease (SD) & $12(24 \%)$ \\
Progressive Disease (PD) & $13(26 \%)$ \\
Overall Response Rate (ORR) & $25(50 \%)$ \\
Clinical Benefit Rate (CBR) & $37(74 \%)$ \\
Median PFS in months (Cl) & $20(17.7-22.2)$ \\
Median OS in months(Cl) & $61(51.4-70.5)$ \\
\hline
\end{tabular}


Survival Function

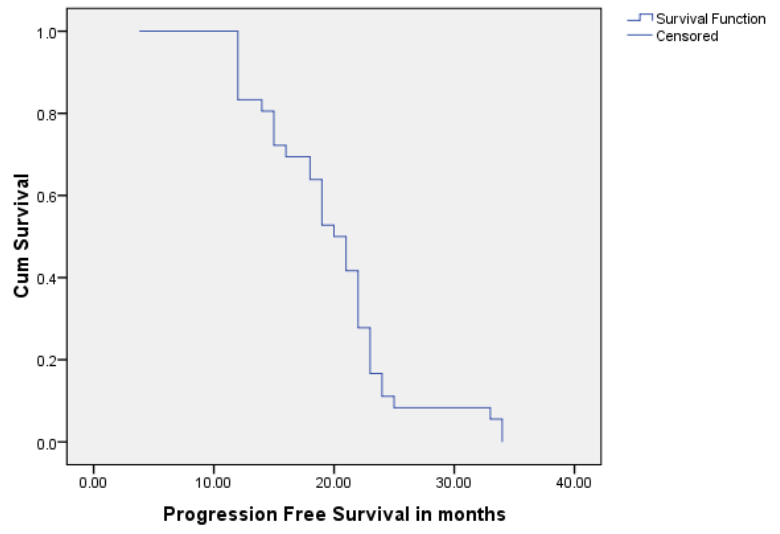

Fig 1. Kaplan-Meier curve of Progression free survival.

Survival Function

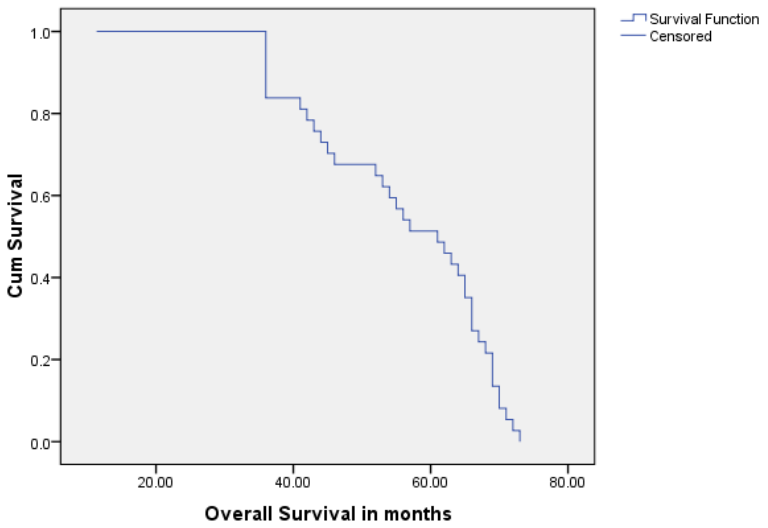

Fig. 2. Kaplan-Meier curve of Overall survival.

Factors associated with better ORR and CBR

Pre-menopausal patients at the start of continuous treatment and of normal BMI with a low or intermediate Ki-67\% index and for the double or one negative hormone with N0-N2 of Grade I-II disease and metastatic single site disease and without brain metastases (Table 6).

\section{Discussion}

Recurrent and MBC have been consistently considered an incurable disease. However, treatment directed against human epidermal growth factor receptor-2 (her-2) significantly improves survival in those patients, and some patients may respond with prolonged complete remission. A consolidated set of clinical trials has led to worldwide approval of trastuzumab as a standard of care for the treatment of patients with locally advanced/metastatic her2-positive breast. ${ }^{21}$

Discontinuation of anti-her2 drugs in recurrent or metastatic settings remains the main unsolved problem, as studies involving $20-25 \%$ of patients with long-term response reported discordant data for patients with prolonged survival who had a break from her2-positive therapies. ${ }^{22}$

In 108 patients with recurrent and metastatic her2-positive breast cancer who received trastuzumab for more than 2 years as first-line treatment, disease progression occurred in 4 of the 27 patients in those stopped trastuzumab. ${ }^{23}$

The ESMO and ASCO guidelines do not currently recommend discontinuing anti-her2 treatments until after several years of sustained complete remission. ${ }^{24,25}$

Maintenance treatment with trastuzumab until disease progression remains the standard after response to first-line chemotherapy and an anti-her 2 agent. The optimal duration of trastuzumab for patients who obtain a complete response is still under debate. Cardiac toxicity is the most significant limiting factor for the use of trastuzumab, although cardiac events are rare and mostly asymptomatic in trastuzumab studies beyond progression, heart attack are and often asymptomatic. ${ }^{26}$

The tolerability of trastuzumab therapy in these patients has also been well established. In September 2016, more than 2.3 million patients were treated with trastuzumab therapy worldwide. However, despite this abundance of clinical experience, there is a paucity of reported data on long-term treatment with trastuzumab. Therefore, the results of our study in patients on long-term therapy with trastuzumab are of considerable interest to the medical oncology community, as many physicians have ongoing questions about the long-term treatment of patients with her2-positive breast cancer locally recurrent/metastatic who are being treated with trastuzumab.

The results of our study suggest that long-term trastuzumab therapy from clinical practice had an acceptable safety profile and was well tolerated. LVEF\% was stable in these patients and in total only 17 serious adverse events were reported, 5 of which were heart-related or led to discontinuation of the treatment.

The risk of serious cardiac adverse events is a concern with trastuzumab treatment. Previous studies have shown a higher incidence of cardiac dysfunction in patients treated with trastuzumab, mainly when trastuzumab treatment was co-administered with chemotherapy drugs and, in particular, in association with anthracycline chemotherapy, in patients with MBC positive for her2. ${ }^{27}$

Other publications investigating long-term treatment with trastuzumab in locally advanced, relapsing or metastatic her2-positive breast cancer have also reported a low risk of serious cardiac adverse events and a continuous response; however, the need remains for additional information on patients with long-term trastuzumab treatment as reported by our study. ${ }^{28}$

In our study, five (10\%) patients had serious adverse cardiac events (marked reduction in LVEF $\%<45 \%$ and developed cardiac toxicity) that were not corrected with supportive treatment and/or postponed treatment, therefore, treatment is permanently suspended. However, 33 (66\%) patients without marked changes in LVEF percentage (range 50-71\%). Although $12(24 \%)$ patients showed a reduction in LVEF\% to less than $50 \%$ (range $47-50 \%$ ) received supportive care and hospitalization encouraged them to complete their treatment, these results suggest that long-term therapy, trastuzumab is feasible and without any additional risk to cardiac safety, particularly with continuous patient care and monitoring in accordance to standard practice.

Long-term tumor remission over several years was achieved in our group of patients who responded to at least 6 months of trastuzumab treatment for local inoperable recurrent or metastatic breast cancer. PFS was 20 months and OS 61 months. 


\begin{tabular}{|c|c|c|c|c|}
\hline Variables & CR no. (\%) & $\begin{array}{c}\text { PR } \\
\text { no.(\%) }\end{array}$ & $\begin{array}{c}\text { SD } \\
\text { no.(\%) }\end{array}$ & $\begin{array}{c}\text { PD } \\
\text { no.(\%) }\end{array}$ \\
\hline \multicolumn{5}{|l|}{ Menopuasal status and sex } \\
\hline Premenapuase patients & $3(6 \%)$ & $9(18 \%)$ & $7(14 \%)$ & $3(6 \%)$ \\
\hline Postmenapuase & $6(12 \%)$ & $5(10 \%)$ & $3(6 \%)$ & $8(16 \%)$ \\
\hline Male & $1(2 \%)$ & $1(2 \%)$ & $2(4 \%)$ & $2(4 \%)$ \\
\hline \multicolumn{5}{|l|}{ Body Mass Index (BMI) } \\
\hline Under Weight [Low BMI $(<18)]$ & $1(2 \%)$ & $2(4 \%)$ & $1(2 \%)$ & $5(10 \%)$ \\
\hline Normal Weight [BM1 $(18-<25)]$ & $7(14 \%)$ & $11(22 \%)$ & $5(10 \%)$ & $4(8 \%)$ \\
\hline Over Weight [High BMI $(\geq 25)]$ & $2(4 \%)$ & $2(4 \%)$ & $6(12 \%)$ & $4(8 \%)$ \\
\hline \multicolumn{5}{|l|}{ Ki67 \% Index status } \\
\hline Low (5\%-15\%) & $5(10 \%)$ & $8(16 \%)$ & $2(4 \%)$ & $2(4 \%)$ \\
\hline Intermediate (16\%-30\%) & $4(8 \%)$ & $6(12 \%)$ & $4(8 \%)$ & $7(14 \%)$ \\
\hline $\operatorname{High}(>30 \%)$ & $1(2 \%)$ & $2(4 \%)$ & $7(14 \%)$ & $4(8 \%)$ \\
\hline \multicolumn{5}{|l|}{ Hormonal Status } \\
\hline $\mathrm{ER}+/ \mathrm{PR}+$ & $0(0 \%)$ & $3(6 \%)$ & $2(4 \%)$ & $5(10 \%)$ \\
\hline$E R+/ P R-$ & $2(4 \%)$ & $5(10 \%)$ & $3(6 \%)$ & $4(8 \%)$ \\
\hline ER-/PR+ & $2(4 \%)$ & $3(6 \%)$ & $4(8 \%)$ & $3(6 \%)$ \\
\hline ER-/PR- & $5(10 \%)$ & $4(8 \%)$ & $3(6 \%)$ & $1(2 \%)$ \\
\hline \multicolumn{5}{|l|}{ Disease Grade } \\
\hline Grade I (well differentiated) & $5(10 \%)$ & $3(6 \%)$ & $5(10 \%)$ & $3(6 \%)$ \\
\hline Grade II (moderate differentiated) & $3(6 \%)$ & $9(18 \%)$ & $6(12 \%)$ & $4(8 \%)$ \\
\hline Grade III (poor differentiated) & $2(4 \%)$ & $3(6 \%)$ & $2(4 \%)$ & $6(12 \%)$ \\
\hline \multicolumn{5}{|l|}{ Tumor size } \\
\hline TO-1 & $2(4 \%)$ & $2(4 \%)$ & $3(6 \%)$ & $3(6 \%)$ \\
\hline $\mathrm{T} 2$ & $2(4 \%)$ & $5(10 \%)$ & $4(8 \%)$ & $4(8 \%)$ \\
\hline T3 & $3(6 \%)$ & $3(6 \%)$ & $5(10 \%)$ & $2(4 \%)$ \\
\hline T4 & $3(6 \%)$ & $6(12 \%)$ & $1(2 \%)$ & $4(8 \%)$ \\
\hline \multicolumn{5}{|l|}{ Nodal state } \\
\hline NO & $5(10 \%)$ & $5(10 \%)$ & $0(0 \%)$ & $1(2 \%)$ \\
\hline N1 & $3(6 \%)$ & $4(8 \%)$ & $3(6 \%)$ & $2(4 \%)$ \\
\hline N2 & $2(4 \%)$ & $4(8 \%)$ & $4(8 \%)$ & $4(8 \%)$ \\
\hline N3 & $0(0 \%)$ & $2(4 \%)$ & $5(10 \%)$ & $6(12 \%)$ \\
\hline \multicolumn{5}{|l|}{ Local Recurrance } \\
\hline Supraclavicular LN & $0(0 \%)$ & $0(0 \%)$ & $2(4 \%)$ & $1(2 \%)$ \\
\hline Axillary LN & $1(2 \%)$ & $0(0 \%)$ & $1(2 \%)$ & $0(0 \%)$ \\
\hline Breast & $0(0 \%)$ & $1(2 \%)$ & $0(0 \%)$ & $0(0 \%)$ \\
\hline Chest Wall & $0(0 \%)$ & $2(4 \%)$ & $0(0 \%)$ & $0(0 \%)$ \\
\hline \multicolumn{5}{|l|}{ Metastatic Disease } \\
\hline Bone only & $3(6 \%)$ & $1(2 \%)$ & $2(4 \%)$ & $1(2 \%)$ \\
\hline Liver only & $4(8 \%)$ & $4(8 \%)$ & $1(2 \%)$ & $0(0 \%)$ \\
\hline Lung only & $2(4 \%)$ & $3(6 \%)$ & $1(2 \%)$ & $1(2 \%)$ \\
\hline Brain only & $0(0 \%)$ & $1(2 \%)$ & $0(0 \%)$ & $0(0 \%)$ \\
\hline Bone with Viscera & $2(4 \%)$ & $2(4 \%)$ & $3(6 \%)$ & $4(8 \%)$ \\
\hline Multiple Viscera & $0(0 \%)$ & $1(2 \%)$ & $2(4 \%)$ & $9(18 \%)$ \\
\hline Decrease EF\% to $47-50 \%$ & $5(10 \%)$ & $4(8 \%)$ & $3(6 \%)$ & $5(10 \%)$ \\
\hline
\end{tabular}

We were able to identify patients who were pre-menopausal at the start of continuous treatment and a normal BMI with a low or intermediate $\mathrm{Ki}-67 \%$ index and double or one negative hormone with Grade I-II N0-N2 disease and single site metastatic disease without brain metastases associated with better ORR and CBR.

Trastuzumab therapy has increased response rates and survival times in recurrent and metastatic settings. ${ }^{29}$ One of the problems of our study is that it is a small sample study of a single arm without a comparative arm, so we have not been able to draw unequivocal conclusions, but we were only able to describe the parameters that influence long-term remission within a highly selected sample.

Another limitation of these analyzes is that recruited patients had previously received trastuzumab treatment and therefore may have had a reduced initial risk of cardiac events and other adverse events in this study. 
This selection means that this study population may not be representative of all trastuzumab-treated breast cancer patients. Therefore, the safety risks reported here may be underestimated compared to the general population, who may need to discontinue trastuzumab treatment early due to serious adverse events or disease worsening. Underestimating safety risks may also be the result of underestimation of serious adverse events or failure to measure LVEF\%, although significant or serious cardiac adverse events or significant decreases in LVEF\% are unlikely to be lost in our study through long-term use with trastuzumab, as patients were regularly monitored.

Finally, there is a paucity of additional information, such as serum and tissue biomarkers, on patients who have performed well with trastuzumab therapy. Some studies have identified predictive biomarkers that could provide a clue to the choice of patients who will benefit most from long-term maintenance of trastuzumab after a complete response to firstline combination therapy. ${ }^{30}$

On the other hand, some other studies have not been able to identify any useful parameter to predict long-term results. ${ }^{31}$ A randomized clinical trial comparing trastuzumab maintenance with only follow-up after a complete response to first-line treatment may be necessary, although the number of patients would be small and the option of discontinuing trastuzumab may not be attractive to at least some patient.

\section{Conclusion}

The results of our study suggest that in patients receiving long-term therapy with trastuzumab for her2-positive disease ( $\geq 5$ years) for patients with inoperable local recurrent/ metastatic breast cancer, treatment with trastuzumab is well tolerated with an acceptable safety that is well tolerated and of good efficacy. Hence, I strongly recommended it. It would be useful to do more research and reports on long-term therapy with trastuzumab. Although the percentage of patients with recurrent and $\mathrm{MBC}$ with long-term tumor remission is low, we provide evidence indicate that positive her- 2 patients who initially respond to trastuzumab treatment possible to achieve long-term tumor remission.

\section{Abbreviation:}

ASCO/CAP guidelines: American Society of Clinical Oncology /College of American Pathologists

AST/ALT: aspartate transaminase/alanine transaminase

CBC : Complete Blood Count

CEP17: Centromere enumeration probe for chromosome 17

ESMO: The European Society for Medical Oncology

HER2: Human epidermal growth factor receptor 2

LVEF: Left ventricular ejection fraction

MBC: Metastatic breast cancer

WHO-PS: World Health. Organization Performance Status

\section{Acknowledgments}

I would like to thank my Colleague Assistant professor Thaer Wally for his assistance with information collection and statistical calculations also thanks for other sub-staff for considerable aid and patient support and finally thanks for our patients accepted to treatment and remained in close follow-up.

\section{Conflict of Interest}

There is absolutely no potential conflict of interest, nor any beneficial relation with drug company.

\section{Ethical Clearance}

Taken from our scientific university committee (Jaber Bin Hayyan Medical University)

\section{References}

1. Mohammeed H. Forouzanfar KJF, Allyne M. Delossantos, Rafael Lozano, Alan D. Lopez, Christopher J. L. Murray, Mohsen Naghavi. Breast and cervical cancer in 187 countries between 1980 and 2010: A systemic analysis. The Lancet 2011;6736:61351-61352.

2. U.S. Breast Cancer Statistics. [Available online at:https://www.breastcancer. org/symptoms/understand_bc/statistics.Final.pdf,cited 25 December 2019].

3. Mastro LD, Lambertini M, Bighin C, et al. Trastuzumab as first-line therapy in her2-positive metastatic breast cancer patients. Expert Rev Anticancer Ther 2012;12:1391-405.

4. Balasubramaniam T. Proposal for the Inclusion of Trastuzumab in the WHO Model List of Essential Medicines for the Treatment of HER2-Positive Breast Cancer. Geneva, Switzerland: Knowledge Ecology International; 2013. [Available online at: http:// www.who.int/selection_medicines/ committees/expert/19/applications/Trastuzumab2_8_2_A_Ad_Final.pdf; cited 4 March 2015]

5. Early Breast Cancer Trialists' Collaborative Group (EBCTCG) (2005) Effects of chemotherapy and hormonal therapy for early breast cancer on recurrence and 15-year survival: An overview of the randomised trials. Lancet 365(9472):1687-1717.

6. Slamon DJ, Godolphin W, Jones LA, et al. Studies of the her-2/neu protooncogene in human breast and ovarian cancer. Science 1989;244:707-12.

7. Baselga J, Cortés J, Kim SB, et al. Pertuzumab plus trastuzumab plus docetaxel for metastatic breast cancer. N Engl J Med 2012;366:109-19.

8. Gobbini E, Ezzalfani M, Dieras V, Delaloge $S$ et al. Time trends of overal survival among metastatic breast cancer patients in the real-life ESME cohort Eur J Cancer 2018:96:17-24.

9. Dawood S, Broglio K, Buzdar AU, Hortobagyi GN, Giordano SH. Prognosis of women with metastatic breast cancer by her 2 status and trastuzumab treatment: An institutionalbased review. J Clin Oncol 2010;28:92-8.

10. National Comprehensive Cancer Network: NCCN clinical practice guidelines in oncology (NCCN guidelines ${ }^{\circledR}$ ): Breast Cancer, V1. 2017. Available at: http://www. nccn.org/professionals/physician_gls/pdf/breast. pdf. Accessed 04 Dec 2017.

11. Swain SM, Baselga J, Kim SB, et al. Pertuzumab, trastuzumab, and docetaxel in HER2-positive metastatic breast Cancer. N Engl J Med 2015;372:724-34.

12. Witzel I, Muller V, Abenhardt W, et al. Long-term tumor remission under trastuzumab treatment for her 2 positive metastatic breast cancer - results from the her-os patient registry. BMC Cancer 2014;14:806.

13. von Minckwitz G, du Bois A, Schmidt M, et al. Trastuzumab beyond progression in human epidermal growth factor receptor 2-positive advanced breast Cancer: A German breast group 26/breast international group 03-05 study. J Clin Oncol 2009;27:1999-2006.

14. Witzel I, Müller V, Abenhardt W, et al. Long-term tumor remission under trastuzumab treatment for HER2 positive metastatic breast cancer-results from the HER-OS patient registry. BMC Cancer 2014;14:806.

15. Cardoso F. 4th ESO-ESMO International Consensus Guidelines for Advanced Breast Cancer (ABC 4). Ann Oncol. 2018:29(8):1634-1657.

16. Giordano SH, Temin S, Chandarlapaty S, Crews JR, Esteva FJ, Kirshner JJ, Krop IE, Levinson J, Lin NU, Modi S, Patt DA, Perlmutter J, Ramakrishna N, Winer EP, Davidson NE. Systemic therapy for patients with advanced human epidermal growth factor receptor 2-positive breast cancer: ASCO clinical practice guideline update. J Clin Oncol 2018;36(26):2736-2740.

17. Tripathy D, Slamon DJ, Cobleigh M, et al. Safety of treatment of metastatic breast cancer with trastuzumab beyond disease progression. J Clin Oncol 2004;22:1063-70

18. Viel E, Arbion F, Barbe C, et al. Prolonged complete response after treatment withdrawal in HER2-overexpressed, hormone receptor negative breast cancer with liver metastases: The prospect of disappearance of an incurable disease. BMC Cancer 2014;14:690

19. Wolff C, Hammond ME et al. Recommendations for human epidermal growth factor receptor 2 testing in breast cancer: American Society of 
Clinical Oncology/College of American Pathologists Clinical Practice Guideline Update. J Clin Oncol. 2013;31(31):3997-4013.

20. Eisenhauer EA, Therasse P, Bogaerts J, Schwartz LH, Sargent D, Ford R, Dancey J, Arbuck S, Gwyther S, Mooney M, Rubinstein L, Shankar L, Dodd L, Kaplan R, Lacombe D, Verweij J. New response evaluation criteria in solid tumours: revised RECIST guideline (version 1.1). Eur J Cancer. 2009 Jan 1:45(2):228-47.

21. National Comprehensive Cancer Network: NCCN clinical practice guidelines in oncology (NCCN guidelines ${ }^{\circledast}$ ): Breast Cancer, V1. 2017. Available at: http://www. nccn.org/professionals/physician_gls/pdf/breast.pdf. Accessed 04 Dec 2017.

22. Daniels B, Kiely BE, Lord SJ, Houssami N, Lu CY, Ward RL, Pearson SA. Longterm survival in trastuzumab-treated patients with HER2-positive metastatic breast cancer: Realworld outcomes and treatment: patterns in a wholeof-population Australian cohort (2001-2016). Breast Cancer Res Treat. 2018;171(1):151-159.

23. Niikura N, Shimomura A, Fukatsu Y et a. Durable complete response in HER2-positive breast cancer: a multicenter retrospective analysis. Breast Cancer Res Treat. 2018:167:81-87.

24. Cardoso F. 4th ESO-ESMO International Consensus Guidelines for Advanced Breast Cancer (ABC 4). Ann Oncol. 2018; 29(8):1634-1657.

25. Giordano SH, Temin S, Chandarlapaty S, Crews JR, Esteva FJ, Kirshner JJ, Krop IE, Levinson J, Lin NU, Modi S, Patt DA, Perlmutter J, Ramakrishna N,
Winer EP, Davidson NE. Systemic therapy for patients with advanced human epidermal growth factor receptor 2-positive breast cancer: ASCO clinical practice guideline update. J Clin Oncol. 2018;36(26):2736-2740.

26. Tripathy D, Slamon DJ, Cobleigh M, et al. Safety of treatment of metastatic breast cancer with trastuzumab beyond disease progression. J Clin Oncol. 2004;22:1063-70

27. Seidman A, Hudis C, Pierri MK, Shak S, Paton V, Ashby M, et al. Cardiac dysfunction in the trastuzumab clinical trials experience. J Clin Oncol. 2002;20:1215-21.

28. Smichkoska S, Lazarova E. Long term trastuzumab in metastatic setting of the patients with HER2 positive breast Cancer. Breast Can Curr Res.2016;1:103.

29. Kiely BE, Soon YY, Tattersall MH, Stockler MR: How long have I got? Estimating typical, best-case, and worst-case scenarios for patients starting first-line chemotherapy for metastatic breast cancer: A systematic review of recent randomized trials. J Clin Oncol 2010;29(4):456-463.

30. Montemurro F, Prat A, Rossi V, et al. Potential biomarkers of long-term benefit from single-agent trastuzumab or lapatinib in her2-positive metastatic breast cancer. Mol Oncol. 2014;8:20-6.

31. Yeo B, Kotsori K, Mohammed K, Walsh G, Smith IE. Long-term outcome of her2 positive metastatic breast cancer patients treated with first-line trastuzumab. Breast 2015;24:751-7. 